Revista Brasileira de Agricultura Irrigada v.10, no.2, p. 515 - 521, 2016

ISSN 1982-7679 (On-line)

Fortaleza, CE, INOVAGRI - http://www.inovagri.org.br

DOI: $10.7127 /$ rbai.v10n200383

Protocolo 383.16 - 04/12/2015 Aprovado em 08/04/2016

\title{
COEFICIENTE DO TANQUE CLASSE A PARA A REGIÃO DE MOSSORÓ, RN
}

Tayd Dayvison Custódio Peixoto'; Sérgio Luiz Aguilar Levien²; Andre Herman Freire Bezerra3; Saulo Tasso Araújo da Silva ${ }^{4}$; José Espínola Sobrinho ${ }^{5}$

\begin{abstract}
RESUMO
O método do Tanque Classe A, para determinar a evapotranspiração de referência (ETo), foi desenvolvido para se ter uma forma prática para estimá-la e aplicar ao manejo da irrigação. Para a realização deste trabalho foram utilizados dados meteorológicos de uma série histórica de 15 anos, no período do ano de 1993 a 2007, da estação climatológica convencional da Universidade Federal Rural do Semi-Árido. Objetivou-se com este trabalho calcular e propor valores médios de coeficientes do Tanque Classe A (Kp) em diferentes intervalos temporais (mensal, sazonal e anual), para a região de Mossoró, Rio Grande do Norte, para auxiliar produtores rurais na sua utilização no manejo da irrigação. Os valores médios mensais do coeficiente $(\mathrm{Kp})$ são os mais recomendados para utilização; a segunda escolha seria a utilização dos valores médios sazonais de 0,79 e 0,62 , para período chuvoso e período seco, respectivamente; e, por último, o valor médio anual, igual a 0,71 .
\end{abstract}

Palavras-chave: dados meteorológicos, evaporação do tanque, evapotranspiração, manejo de irrigação

\section{CLASS A PAN COEFFICIENT TOTHE REGION OF MOSSORÓ, RN, BRAZIL}

\begin{abstract}
The Class A pan method, to determine the reference evapotranspiration (ETo), was developed to be a practical way to estimate it and apply to irrigation management. For this work, a historical meteorological data series of 15 years, within the period of 1993 to 2007, of the conventional climatological station of Universidade Federal Rural do Semi-Árido was used. The objective of this

\footnotetext{
${ }^{1}$ Engenheiro Agrícola e Ambiental, Mestrando, Bolsista CAPES, Programa de Pós-Graduação em Manejo de Solo e Água, Universidade Federal Rural do Semi-Árido (UFERSA), Mossoró, RN; e-mail: dayvisonpeixoto@ hotmail.com

${ }^{2}$ Engenheiro Agrícola, D.Sc., Programa de Pós-Graduação em Irrigação e Drenagem, UFERSA, Mossoró, RN, e-mail: sergiolevien@ufersa.edu.br

${ }^{3}$ Engenheiro Agrônomo, M.Sc., Doutorando, Bolsista CNPq., Programa de Pós-Graduação em Engenharia de Sistemas Agrícolas, ESALQ/USP, Piracicaba, SP, e-mail: andre.herman@yahoo.com

${ }^{4}$ Engenheiro Agrônomo, D.Sc., Departamento de Ciências Ambientais e Tecnológicas, UFERSA, Mossoró, RN, e-mail: saulo@ufersa.edu.br

${ }^{5}$ Engenheiro Agrônomo, D.Sc., Departamento de Ciências Ambientais e Tecnológicas, UFERSA, Mossoró, RN, e-mail: jespinola@ufersa.edu.br
} 
work is to calculate and propose average values of Class A pan coefficient $(\mathrm{Kp})$ at different time intervals (monthly, seasonal and annual), for the region of Mossoró, Rio Grande do Norte, Brazil, in order to assist farmers on its use in irrigation management. The monthly average values of the coefficient $(\mathrm{Kp})$ are the most recommended for use; the second choice would be the use of the seasonal mean values of 0.79 and 0.62 for rainy season and dry season, respectively; and, for last, the annual average value, equal to 0.71 .

Keywords: meteorological data, pan evaporation, evapotranspiration, irrigation management

\section{INTRODUÇÃO}

A preocupação mundial com os recursos hídricos e, consequentemente, com a quantidade de água utilizada na irrigação resultam em uma busca por um manejo racional da irrigação, que seja mais eficaz, que utilize somente o que é realmente necessário para obter altas produtividades e não desperdiçar água de boa qualidade, principalmente na região Nordeste do Brasil que possui limitados recursos hídricos devido ao inconstante regime de chuvas (PEIXOTO, 2011).

Para um manejo eficiente da irrigação, qualquer que seja a cultura, é essencial a determinação da evapotranspiração; e por outro lado, quanto mais precisa for essa determinação, melhor será a quantificação das doses de irrigação a serem aplicadas (KISI, 2006; CARVALHO et al., 2007).

A evapotranspiração da cultura (ETc) é um fator que deve ser levado em consideração quando se pensa em irrigação, pois poderemos otimizar o uso da água. A irrigação exige grande demanda de água e esta não pode ser usada em excesso nem de maneira escassa para não haver desperdício ou redução da produtividade das culturas agrícolas. A utilização eficiente da água está se tornando cada vez mais importante devido à escassez de recursos hídricos na região Nordeste e ao elevado custo da energia, o que torna cada vez mais necessário o uso de metodologias apropriadas ao manejo racional do uso da água.

Para manejar corretamente uma cultura deve-se determinar com precisão suas necessidades hídricas, e para tal, tem que se considerar que o clima, as características da cultura, o manejo e o meio de desenvolvimento são fatores que afetam a evaporação e a transpiração, que somadas são chamadas de evapotranspiração (BEZERRA, 2009).

Existem vários métodos para determinação da evapotranspiração de referência (ETo), como lisímetros, tabelas e equações, onde geralmente na determinação ou ajuste de valores de evaporação do tanque para obtenção da taxa de evaporação ou evapotranspiração de referência usa-se um coeficiente empírico, Kp, tanto para aplicações na agricultura quanto na hidrologia (MOLINA MARTÍNEZ et al., 2006).

A ETo, calculada com base em dados do Tanque Classe A, é um método indireto e bastante utilizado no Brasil devido a sua facilidade no uso e baixo custo. $\mathrm{O}$ mesmo necessita da evaporação do Tanque Classe A (ECA) e do coeficiente do tanque (Kp), que varia de acordo com as condições climáticas e locais, para ser estimada (SENTELHAS; FOLEGATTI, 2003; MENDONÇA et al., 2006). É comum a adoção de um valor fixo de $\mathrm{Kp}$ quando dados de umidade relativa e velocidade do vento não são disponíveis (PEREIRA et al., 2002).

Os valores de Kp podem ser calculados (ou estimados) usando equações propostas por alguns autores na literatura como Cuenca (1989), Snyder (1992), Pereira et al. (1995) e Orang (1998), ou por tabelas ou equações propostas pela $\mathrm{FAO}$ em seus manuais FAO24 (DOORENBOS; PRUITT, 1977) e FAO56 (ALLEN et al., 1998), ou modelos baseados em Redes Neurais Artificiais, com técnicas de Algoritmos Genéticos ou Lógica Fuzzy, por exemplo, trabalhos de Kisi (2006) e Guven e Kisi (2011), ou diretamente relacionando o valor da evaporação do Tanque Classe A obtido com o valor da evapotranspiração de referência estimado através da equação FAO PenmanMonteith (ALLEN et al., 1998).

Objetivou-se com este trabalho estimar valores de Kp para a região de Mossoró, Rio Grande do Norte, utilizando dados de ECA 


\section{COEFICIENTE DO TANQUE CLASSE A PARA A REGIÃO DE MOSSORÓ, RN}

registrados comparando-os com dados de ETo estimados pelo método FAO Penman-Monteith, considerado padrão, usando dados de uma série histórica de 15 anos (de 1993 a 2007), e propor valores médios de Kp mensais, sazonais (período seco e período chuvoso) e anual, para auxiliar a utilização, por produtores da região estudada que não dispõem de dados meteorológicos, no manejo da irrigação.

\section{MATERIAL E MÉTODOS}

Nesse trabalho foram utilizados dados meteorológicos de uma série histórica de 15 anos, no período de 1993 a 2007, da Estação Climatológica Convencional da Universidade Federal Rural do Semi-Árido (UFERSA), no município de Mossoró (5'12'48' S; 37²18'43', W; 18m), Rio Grande do Norte.

Para cálculo da evapotranspiração de referência (ETo), pelo método FAO PenmanMonteith (ALLEN et al., 1998), equação que tem sido usada quando dados de lisímetros não estão disponíveis (GAVILAN et al., 2006; LEE; CHO, 2012), foi utilizado o software SEEVA (BEZERRA, 2009). Este software é uma ferramenta de estimativa da ETo, que, dentre outras funções, permite estimar a mesma por cinco diferentes métodos, entre eles o método FAO Penman-Monteith, que é representado pela equação:

$$
\mathrm{ETo}=\frac{0,408 \cdot \Delta \cdot(\mathrm{Rn}-\mathrm{G})+\gamma \cdot\left(\frac{900 \cdot \mathrm{U}_{2}}{\mathrm{~T}+273}\right) \cdot\left(\mathrm{e}_{\mathrm{s}}-\mathrm{e}_{\mathrm{a}}\right)}{\Delta+\gamma \cdot\left(1+0,34 \cdot \mathrm{U}_{2}\right)}
$$

em que ETo é a evapotranspiração de referência $\left(\mathrm{mm} \mathrm{dia}^{-1}\right) ; \Delta$ é a declinação da curva de saturação de vapor d'água $\left(\mathrm{kPa}{ }^{\circ} \mathrm{C}^{-1}\right)$; $\mathrm{Rn}$ é a radiação líquida na superfície da cultura $\left(\mathrm{MJ} \mathrm{m}^{-2} \mathrm{dia}^{-1}\right) ; \mathrm{G}$ é a densidade de fluxo de calor no solo $\left(\mathrm{MJ} \mathrm{m}^{-2} \mathrm{dia}^{-}\right.$ $\left.{ }^{1}\right)$; $\gamma$ é a constante psicrométrica $\left(\mathrm{kPa}^{\circ} \mathrm{C}^{-1}\right) ; \mathrm{U}_{2}$ é a velocidade do vento a $2 \mathrm{~m}$ de altura $\left(\mathrm{m} \mathrm{s}^{-1}\right)$; $\mathrm{T}$ é a temperatura média diária do ar a $2 \mathrm{~m}$ de altura $\left({ }^{\circ} \mathrm{C}\right)$; e é é a pressão de saturação do vapor $(\mathrm{kPa})$; e $\mathrm{e}_{\mathrm{a}}$ é a pressão de vapor atual $(\mathrm{kPa})$.

Os valores de $\mathrm{Kp}$ foram obtidos através da seguinte relação:

$$
\mathrm{Kp}=\frac{\mathrm{ETo}}{\mathrm{ECA}}
$$

em que Kp é o coeficiente do Tanque Classe A; ETo é a evapotranspiração de referência calculada pelo método FAO Penman-Monteith; e ECA é a evaporação do Tanque Classe A registrada na estação.

A relação anterior deriva da equação principal de obtenção da ETo a partir de dados do Tanque Classe A, como mostrado a seguir:

$$
\mathrm{ETo}=\mathrm{Kp} \cdot \mathrm{ECA}
$$

Os valores foram calculados como médias mensais, médias sazonais (período chuvoso, de janeiro a junho, e período seco, de julho a dezembro) e média anual.

Também se comparou os valores de Kp obtidos com dados de um trabalho recente de Peixoto et al. (2014) realizado com o mesmo banco de dados, comparando valores de ETo estimados baseados nos valores de evaporação de Tanque Classe A, e utilizando diferentes métodos propostos na literatura.

Para se avaliar o desempenho dos valores estimados de $\mathrm{Kp}$, baseados em dados do tanque Classe A, foram utilizados alguns índices estatísticos que preconizam um estudo de análise de regressão linear: soma dos quadrados médios (SQM), erro absoluto médio (EAM), erro relativo médio (ERM), e índice de concordância (d) proposto por Willmott (1981), os quais são dados pelas seguintes equações:

$$
\mathrm{SQM}=\sqrt{\frac{1}{\mathrm{n}} \cdot \sum\left(\mathrm{E}_{\mathrm{i}}-\mathrm{O}_{\mathrm{i}}\right)^{2}}
$$

$$
\begin{gathered}
E A M=\frac{1}{n} \cdot \sum\left|E_{i}-O_{i}\right| \\
E R M=\frac{1}{n} \cdot \sum\left(\frac{E_{i}-O_{i}}{O_{i}}\right) \\
d=1-\frac{\sum\left(\mathrm{E}_{\mathrm{i}}-\mathrm{O}_{\mathrm{i}}\right)^{2}}{\sum\left(\left|\mathrm{E}_{\mathrm{i}}-\overline{\mathrm{O}}\right|+\left|\mathrm{E}_{\mathrm{i}}-\overline{\mathrm{O}}\right|\right)^{2}}
\end{gathered}
$$


em que $E_{i}$ corresponde ao valor estimado pelo modelo; $\mathrm{O}_{\mathrm{i}}$ corresponde ao valor observado experimentalmente (ou estimado pelo modelo considerado padrão); e $\overline{\mathrm{O}}$ é o valor médio entre os dados observados.

\section{RESULTADOS E DISCUSSÃO}

Grande número de produtores da região não possui acesso a dados meteorológicos, sendo assim que, através da ETo, estimada pelo método FAO Penman-Monteith, e de dados de evaporação do Tanque Classe A, foram determinados valores de $\mathrm{Kp}$ médios mensais, sazonais e anual, com base em dados da série histórica de 15 anos, de 1993 a 2007, obtidos na
Estação Climatológica Convencional da Universidade Federal Rural do Semi-Árido, no Campus Central em Mossoró, RN.

Os valores médios do coeficiente do tanque (Kp) foram obtidos através da equação (2) e são apresentados na Tabela1. Os índices de desempenho dos valores estimados como média anual e médias sazonais em relação às médias mensais são apresentados na Tabela 2 .

Baseado nas Tabelas 1 e 2 e observando a Figura 1, pode-se deduzir que o melhor é usar os valores médios mensais, em primeiro lugar; os valores médios sazonais, como segunda opção; e, finalmente, como última opção usar um valor médio anual.

Tabela 1. Valores médios de Kp mensais, sazonais e anual para a região de Mossoró, RN

\begin{tabular}{cccc}
\multirow{2}{*}{ Período } & \multicolumn{3}{c}{ Kp } \\
\cline { 2 - 4 } & Mensal & Anual & Sazonal \\
\hline Janeiro & 0,71 & 0,71 & 0,79 \\
Fevereiro & 0,78 & 0,71 & 0,79 \\
Março & 0,84 & 0,71 & 0,79 \\
Abril & 0,87 & 0,71 & 0,79 \\
Maio & 0,82 & 0,71 & 0,79 \\
Junho & 0,73 & 0,71 & 0,79 \\
Julho & 0,61 & 0,71 & 0,62 \\
Agosto & 0,62 & 0,71 & 0,62 \\
Setembro & 0,62 & 0,71 & 0,62 \\
Outubro & 0,62 & 0,71 & 0,62 \\
Novembro & 0,62 & 0,71 & 0,62 \\
Dezembro & 0,65 & 0,71 & 0,62 \\
\hline
\end{tabular}

Tabela 2. Valores dos índices de desempenho

\begin{tabular}{ccccc}
\hline \multirow{2}{*}{ Kp } & \multicolumn{4}{c}{ Índice } \\
\cline { 2 - 5 } & SQM & EAM & ERM & d \\
\hline Anual & 0,0939 & 0,0842 & 0,1189 & 0,0455 \\
Sazonal & 0,0417 & 0,0292 & 0,0382 & 0,9426 \\
\hline
\end{tabular}


COEFICIENTE DO TANQUE CLASSE A PARA A REGIÃo DE MOSSORÓ, RN

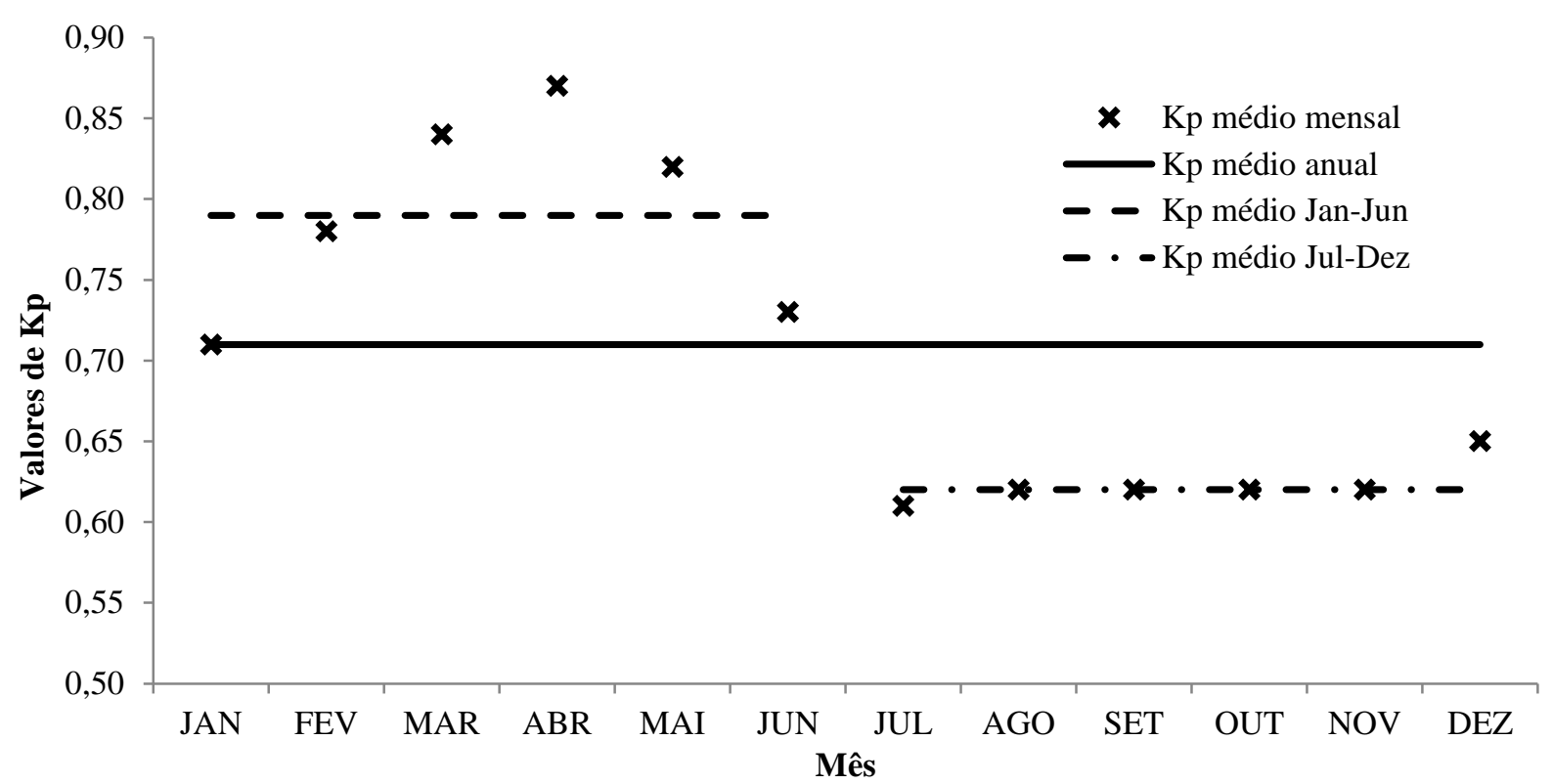

Figura1. Comportamento dos valores de Kp ao longo do ano na região de Mossoró, RN

Observa-se que durante os meses mais secos do ano (julho a dezembro), período em que se faz o uso da irrigação com mais frequência, existe uma constância nos valores de Kp a partir do mês de julho. Assim, um valor fixo de Kp, para o referido período, igual a 0,62 pode ser recomendado. Fazendo-se uso deste coeficiente fixo percebe-se que o maior erro em relação a outros meses do período seco será de aproximadamente $4 \%$.

No período mais chuvoso na região (janeiro a junho), época em que o uso da irrigação é menor em relação ao restante do ano, faz-se uso de irrigação suplementar para suprir a necessidade hídrica das culturas. Um valor médio de Kp, para o período, é igual a 0,79 , mas o uso deste valor pode resultar em erros por volta de $11 \%$.

$\mathrm{O}$ valor médio anual para o coeficiente do Tanque Classe A é igual a 0,71, mas este valor pode resultar em erros de até $18 \%$, subestimando os valores no período chuvoso e superestimando no período seco, como observado na Figura 1.

Comparou-se, também, os dados obtidos para $\mathrm{Kp}$, utilizando a metodologia proposta neste trabalho, com dados obtidos por Peixoto et al. (2014), os quais são apresentados na Tabela 2.

Tabela 2. Valores de Kp médios mensais, sazonais e anual para a região de Mossoró, RN, comparados com valores obtidos por Peixoto et al. (2014) utilizando diferentes metodologias

\begin{tabular}{c|cccccccc}
\hline Período & $\begin{array}{c}\text { FAO56 } \\
\text { PM }\end{array}$ & Cuenca & Snyder & Pereira & Orang & $\begin{array}{c}\text { FAO56 } \\
(\text { FAO } \\
\text { A) }\end{array}$ & $\begin{array}{c}\text { FAO24 } \\
\text { Mensal }\end{array}$ & $\begin{array}{c}\text { FAO24 } \\
\text { Anual }\end{array}$ \\
\hline Janeiro & 0,71 & 0,65 & 0,72 & 0,64 & 0,65 & 0,68 & 0,75 & 0,70 \\
Fevereiro & 0,78 & 0,67 & 0,74 & 0,65 & 0,67 & 0,70 & 0,75 & 0,70 \\
Março & 0,84 & 0,70 & 0,79 & 0,68 & 0,71 & 0,73 & 0,75 & 0,70 \\
Abril & 0,87 & 0,73 & 0,82 & 0,72 & 0,73 & 0,75 & 0,75 & 0,70 \\
Maio & 0,82 & 0,71 & 0,80 & 0,70 & 0,72 & 0,74 & 0,75 & 0,70 \\
Junho & 0,73 & 0,69 & 0,76 & 0,68 & 0,69 & 0,72 & 0,75 & 0,70 \\
Julho & 0,61 & 0,67 & 0,73 & 0,66 & 0,67 & 0,70 & 0,70 & 0,70 \\
Agosto & 0,62 & 0,62 & 0,67 & 0,63 & 0,62 & 0,65 & 0,70 & 0,70 \\
Setembro & 0,62 & 0,60 & 0,64 & 0,60 & 0,60 & 0,63 & 0,60 & 0,70 \\
Outubro & 0,62 & 0,59 & 0,63 & 0,59 & 0,59 & 0,62 & 0,60 & 0,70 \\
Novembro & 0,62 & 0,60 & 0,65 & 0,60 & 0,60 & 0,63 & 0,60 & 0,70 \\
Dezembro & 0,65 & 0,61 & 0,66 & 0,61 & 0,62 & 0,64 & 0,60 & 0,70 \\
\hline
\end{tabular}


Peixoto et al.

\begin{tabular}{c|cccccccc}
\hline Jan-Jun & 0,79 & 0,69 & 0,77 & 0,68 & 0,70 & 0,72 & 0,75 & 0,70 \\
Jul-Dez & 0,62 & 0,62 & 0,66 & 0,61 & 0,62 & 0,65 & 0,63 & 0,70 \\
\hline Anual & 0,71 & 0,65 & 0,72 & 0,65 & 0,66 & 0,68 & 0,69 & 0,70 \\
\hline
\end{tabular}

Fonte: os autores e dados adaptados de Peixoto et al. (2014)

Observando-se os dados obtidos por Peixoto et al. (2014) nota-se que os valores mensais obtidos usando diferentes metodologias propostas subestimam, em média, em até $9 \%$, com exceção dos métodos de Snyder e FAO24 anual, que superestimam em $2 \%$ e $1 \%$, respectivamente.

\section{CONCLUSÃO}

Os valores médios mensais do coeficiente do tanque Classe A (Kp) são os mais recomendados para a utilização por produtores sem acesso a dados climatológicos. A segunda escolha seria a utilização dos valores médios sazonais de 0,79 e 0,62 , para período chuvoso e seco, respectivamente e, por último, o valor médio anual, igual a 0,71 .

\section{REFERÊNCIAS}

ALLEN, R.G.; PEREIRA, L.S.; RAES, D.; SMITH, M. Crop evapotranspiration: guidelines for computing crop water requirements. Rome: FAO, 1998, 300p. (FAO, Irrigation and Drainage Paper, 56).

BEZERRA, A.H.F. Software de simulação do coeficiente de cultura. 2009. 60f. Monografia (Graduação em Agronomia), Universidade Federal Rural do Semi-Árido, Mossoró, 2009. Disponível em: http://ebiblio.ufersa.edu.br/Download/18898.pdf >. Acessado em: 25 jul 2012.

CARVALHO, L.C.C.; BEZERRA, F.M.L. CARVALHO, M.A.R. Evapotranspiração e coeficientes de cultivo da melancia sem sementes. Revista Ciência Agronômica, Fortaleza, v.39, n.1, p.53-59, 2007.

CUENCA, R.H. Irrigation system design: an engineering approach. New Jersey: Prentice-Hall, Englewood Cliffs. 1989, 133p.
DOORENBOS, J.; PRUITT, W.O. Crop water requirements. Rome: FAO, 1977, 168 p. (FAO, Irrigation and Drainage Paper, 24).

GAVILAN, P.; LORITE, I.J.; TORNERO, S.; BERENGENA, J. Regional calibration of Hargreaves equation for estimating reference ET in a semiarid environment. Agricultural Water Management, v.81, n.3, p.257-281, 2006.

GUVEN, A.; KISI, O. Daily pan evaporation modeling using linear genetic programming technique. Irrigation Science, v.29, p.135-145, 2011

KISI, O. Daily pan evaporation modelling using a neuro-fuzzy computing technique. Journal of Hydrology, v.329, n.3, p.636-646, 2006.

LEE, K.; CHO, H. Simple method for estimating pan coefficient: conversion of pan evaporation to reference evapotranspiration. Journal of Irrigation and Drainage Engineering, v.138, n.1, p.98-103, 2012.

MENDONÇA, J.C.; SOUSA, E.F.; ANDRE, R.G.B.; BERNARDO, S. Coeficientes do tanque Classe "A" para a estimativa da evapotranspiração de referência, em Campos dos Goytacazes, RJ. Revista Brasileira de Agrometeorologia, Piracicaba, v.14, n.1, p.123128, 2006.

MOLINA MARTÍNEZ, J.M.; MARTÍNEZ ALVAREZ, V.; GONZÁLEZ-REAL, M.M.; BAILLE, A. A simulation model for predicting hourly pan evaporation from meteorological data. Journal of Hydrology, v.318, n.1, p.250-261, 2006.

ORANG, M. Potential accuracy of the popular non-linear regression equations for estimating pan coefficient values in the original and FAO-24 tables. Sacramento: California Department of Water Resources, 1998, 68p. 
PEIXOTO, T.D.C. Evapotranspiração de referência estimada com diferentes metodologias de uso do tanque Classe A. 2011. 33f. Monografia (Graduação em Engenharia Agrícola e Ambiental), Universidade Federal Rural do SemiÁrido, Mossoró, 2011. Disponível em: < http://ebiblio.ufersa.edu.br/Download/22456.pdf >. Acessado em: 07 jul 2012.

PEIXOTO, T.D.C.; LEVIEN, S.L.A.; BEZERRA, A.H.F.; ESPÍNOLA SOBRINHO, J. Avaliação de diferentes metodologias de estimativa da ETo baseadas no Tanque Classe A, em Mossoró, RN. Revista Caatinga, Mossoró, v.27, n.2, p.58-65, 2014.

PEREIRA, A.R; VILLA NOVA, N.A.; PEREIRA, A.S.; BARBIERI, V. A model for the class A pan coefficient. Agricultural and Forest Meteorology, v.76, n.1, p.75-82, 1995.
PEREIRA, A.R.; ANGELOCCI, L.R.; SENTELHAS, P.C. Agrometeorologia: Fundamentos e aplicações práticas. Guaíba: Agropecuária, 2002, 478p.

SENTELHAS P.C.; FOLEGATTI, M.V. Class A pan coefficients $(\mathrm{Kp})$ to estimate daily reference evapotranspiration (ETo). Revista Brasileira de Engenharia Agrícola e Ambiental, Campina Grande, v.7, n.1, p.111-115, 2003.

SNYDER, R.L. Equation for evaporation pan to evapotranspiration conversion. Journal of Irrigation and Drainage Engineering, v.118, n.6, p.977-980, 1992.

WILLMOTT, C.J. On the validation of models. Physical Geography, v.2, n.2, p.184-194, 1981. 